Molecules 2003, 8, 735-743

molecules

ISSN 1420-3049

http://www.mdpi.org/

\title{
Novel 4-Aroyl-3-alkoxy-2(5H)-furanones as Precursors for the Preparation of Furo[3,4-b][1,4]-diazepine Ring System
}

\author{
Hans Zimmer ${ }^{1, \dagger}$, Christian P. Librera ${ }^{1}$, Sven Hausner ${ }^{1}$, Jeanette Bauer ${ }^{1}$ and Adel Amer ${ }^{2, *}$ \\ ${ }^{1}$ Department of Chemistry, University of Cincinnati, Cincinnati, OH 45221-0172, U.S.A. \\ ${ }^{2}$ Department of Chemistry, Faculty of Science, Alexandria University, Alexandria, Egypt, Tel.: (+20) \\ 12221 9463; fax: (+20) 34911794
}

* Author to whom correspondence should be addressed; e-mail: $\underline{\text { amerkishk@yahoo.com }}$

$\$$ Part 16 of the series Synthetic Reactions and Structural Studies of Heterocycles Containing Nitrogen; for part 15 see [1].

${ }^{\dagger}$ Deceased June 2001

Received: 29 April 2003; in revised form: 17 September 2003 / Accepted 18 September 2003 / Published: 31 October 2003

\begin{abstract}
A general synthesis of tetronic acid derivatives, namely 4-aroyl-3-alkoxy-2(5H)furanones, is achieved via the treatment of an anhydrous dimethylformamide (DMF) solution of 4-aroyl-3-hydroxy-2(5H)-furanones with 1,8-diazabicyclo[5.4.0]undec-7-ene (DBU) as base at $-10-0^{\circ} \mathrm{C}$, followed by the addition of alkyl iodide. Their structural assignments are based on spectroscopic data and confirmed by X-ray crystallography. These furanones were used as starting materials for the preparation of furodiazepines.
\end{abstract}

Keywords: 4-Aroyl-3-alkoxy-2(5H)-furanones, 7-aryl-4,5-dihydro-2-oxo-3H,8H-furo[3,4-b][1,4]diazepines, X-ray structures. 


\section{Introduction}

Benzodiazepines are an important class of psychotherapeutic compounds. In recent years some examples of heterocyclic rings fused to the seven member diazepine ring system have been synthesized which exhibit psychotropic activities [2-7]. Recently, we have reported on a facile synthesis of novel furodiazepines, namely 7-aryl-4,5-dihydro-2-oxo-3H,8H-furo[3,4-b][1,4]diazepines (1) using 4-aroyl3-methoxy-2(5H)-furanones (2) [1,8]. This procedure was however limited since many 4-aroyl-3hydroxy-2(5H)-furanones $3[9,10]$ are insoluble in ether, the solvent needed for the transformation of $\mathbf{3}$ into the 3-methoxy analogs $2\left(\mathrm{R}=\mathrm{CH}_{3}\right)($ Scheme 1$)$.

Scheme 1.<smiles>[R]OC1=C(C(=O)[Al]C2CCN(C)C2)COC1=O</smiles>

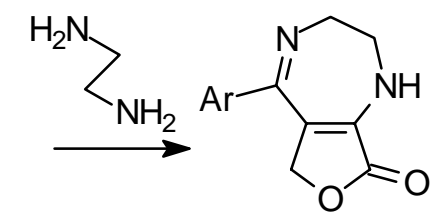

1

\section{Results and Discussion}

In this work we report the results based on our efforts to develop an extended, general O-alkylation procedure for compounds of type $\mathbf{3}$. We found that treatment of a solution of $\mathbf{3}$ in anhydrous dimethylformamide (DMF) with 1,8-diazabicyclo[5.4.0] undec-7-ene (DBU) as base at $-10-0^{\circ} \mathrm{C}$, followed by the addition of a primary alkyl iodide afforded type $\mathbf{2}$ compounds in moderate yields (Scheme 2). Our results are summarized in Table 1

\section{Scheme 2}

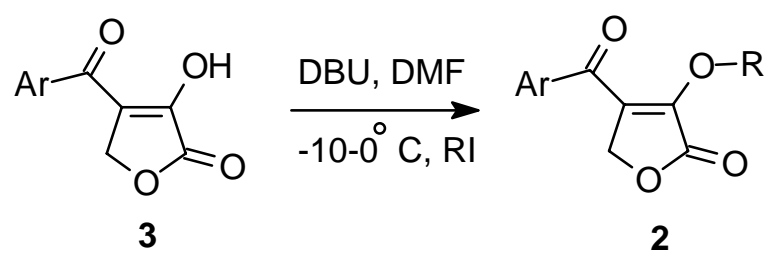


Table 1.

\begin{tabular}{|c|c|c|c|c|}
\hline Entry & Ar & $\mathbf{R}$ & $\begin{array}{c}\text { Yield } \\
(\%)\end{array}$ & $\begin{array}{l}\text { Mp } \\
\left({ }^{\circ} \mathbf{C}\right)\end{array}$ \\
\hline $2 \mathbf{a}$ & $\mathrm{C}_{6} \mathrm{H}_{5}$ & $\mathrm{CH}_{3} \mathrm{CH}_{2-}$ & 42 & Oil \\
\hline $2 b$ & $\mathrm{C}_{6} \mathrm{H}_{5}$ & $\mathrm{CH}_{2}=\mathrm{CHCH}_{2}-$ & 51 & 52 \\
\hline $2 c$ & $o-\mathrm{Cl} \mathrm{C}{ }_{6} \mathrm{H}_{4}$ & $\mathrm{CH}_{3} \mathrm{CH}_{2^{-}}$ & 35 & 48 \\
\hline 2d & $o-\mathrm{Cl} \mathrm{C}{ }_{6} \mathrm{H}_{4}$ & $\left(\mathrm{CH}_{3}\right)_{2} \mathrm{CHCH}_{2-}$ & 41 & Oil \\
\hline $2 e$ & $m-\mathrm{CNC}_{6} \mathrm{H}_{4}$ & $\mathrm{CH}_{3-}$ & 47 & 116 \\
\hline $2 f$ & $p-\mathrm{CH}_{3} \mathrm{C}_{6} \mathrm{H}_{4}$ & $\mathrm{CH}_{3-}$ & 31 & Oil \\
\hline $2 \mathrm{~g}$ & $p-\mathrm{CH}_{3} \mathrm{C}_{6} \mathrm{H}_{4}$ & $\mathrm{CH}_{3} \mathrm{CH}_{2-}$ & 28 & Oil \\
\hline $2 \mathrm{~h}$ & $p-\mathrm{CH}_{3} \mathrm{C}_{6} \mathrm{H}_{4}$ & $\mathrm{CH}_{3}\left(\mathrm{CH}_{2}\right)_{2} \mathrm{CH}_{2-}$ & 29 & 42 \\
\hline $2 \mathbf{i}$ & $p-\mathrm{CH}_{3} \mathrm{C}_{6} \mathrm{H}_{4}$ & $\mathrm{CH}_{3}\left(\mathrm{CH}_{2}\right)_{5} \mathrm{CH}_{2-}$ & 31 & Oil \\
\hline $2 \mathbf{j}$ & $p-\mathrm{CH}_{3} \mathrm{C}_{6} \mathrm{H}_{4}$ & $\mathrm{C}_{6} \mathrm{H}_{5} \mathrm{CH}_{2^{-}}$ & 42 & 84 \\
\hline $2 k$ & $p-\mathrm{CH}_{3} \mathrm{C}_{6} \mathrm{H}_{4}$ & $\mathrm{CH}_{2}=\mathrm{CHCH}_{2^{-}}$ & 48 & 70 \\
\hline 21 & $p-\mathrm{CH}_{3} \mathrm{C}_{6} \mathrm{H}_{4}$ & $\left(\mathrm{CH}_{3}\right)_{2} \mathrm{CHCH}_{2-}$ & 17 & 55 \\
\hline $2 \mathrm{~m}$ & $p-\mathrm{CH}_{3} \mathrm{C}_{6} \mathrm{H}_{4}$ & $\mathrm{CH}_{3} \mathrm{CH}_{2} \mathrm{O}_{2} \mathrm{CH}_{2}-$ & 53 & 84 \\
\hline $2 n$ & $p-\mathrm{CH}_{3} \mathrm{C}_{6} \mathrm{H}_{4}$ & $\left(\mathrm{CH}_{3}\right)_{2} \mathrm{CH}-$ & 23 & 73 \\
\hline 20 & $p-\mathrm{CH}_{3} \mathrm{C}_{6} \mathrm{H}_{4}$ & $\left(\mathrm{CH}_{2}\right)_{4} \mathrm{CH}-$ & 25 & 80 \\
\hline $2 p$ & $p-\mathrm{NO}_{2} \mathrm{C}_{6} \mathrm{H}_{4}$ & $\mathrm{CH}_{3^{-}}$ & 30 & 85 \\
\hline $2 q$ & 5- $\mathrm{CH}_{3}$-2-thienyl & $\mathrm{CH}_{3-}$ & 33 & Oil \\
\hline 4 & $p-\mathrm{CH}_{3} \mathrm{C}_{6} \mathrm{H}_{4}$ & $o-\mathrm{C}_{6} \mathrm{H}_{4}\left(\mathrm{CH}_{2^{-}}\right)_{2}$ & 24 & 98 \\
\hline
\end{tabular}

This O-alkylation could also be employed to synthesize bis-ethers. Thus, when one equivalent of 1,2-bis(iodomethyl)benzene was allowed to react with two equivalents of $3\left(\mathrm{Ar}=4-\mathrm{CH}_{3} \mathrm{C}_{6} \mathrm{H}_{4}\right)$, 4-(methylbenzoyl)-3-[2-(4-methylbenzoyl)-2-oxo(3-hydrofuryl-5-oxy)methylphenyl)methoxy]-(5H)furan-2-one (4) was obtained in $24 \%$ yield.

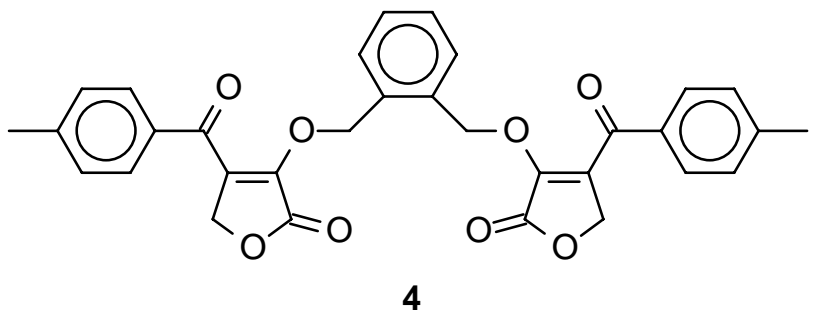

Depending upon the reactivity of the alkyl iodide an excess ranging between one to five equivalents was used to favor the formation of the corresponding ether 2 in an SN2-like reaction. Secondary alkyl iodide were also could be used in this reaction. Structural assignments of the ethers 2 are made on spectroscopic ground, which are summarized in Table 2. Definite proof of the ether 
structures 2 was obtained by X-ray analyses[11]. X-ray structures were obtained for the compounds 2h $\left(\mathrm{R}=\mathrm{CH}_{3}\left(\mathrm{CH}_{2}\right)_{2} \mathrm{CH}_{2}-\right), \mathbf{2 j}\left(\mathrm{R}=\mathrm{C}_{6} \mathrm{H}_{5} \mathrm{CH}_{2^{-}}\right)$, 2k $\left(\mathrm{R}=\mathrm{CH}_{2}=\mathrm{CHCH}_{2^{-}}\right), \mathbf{2 l}\left(\mathrm{R}=\left(\mathrm{CH}_{3}\right)_{2} \mathrm{CHCH}_{2^{-}}\right)$and $\mathbf{2 0}$ $\left(\mathrm{R}=\left(\mathrm{CH}_{2}\right)_{4} \mathrm{CH}-\right)$. Figure 1 shows the $\mathrm{X}$-ray structure of $\mathbf{2} \mathbf{k}$ as a prototype.

Figure 1. X-ray structure of compound $\mathbf{2 k}\left(\mathrm{R}=\mathrm{CH}_{2}=\mathrm{CHCH}_{2}^{-}\right)$.

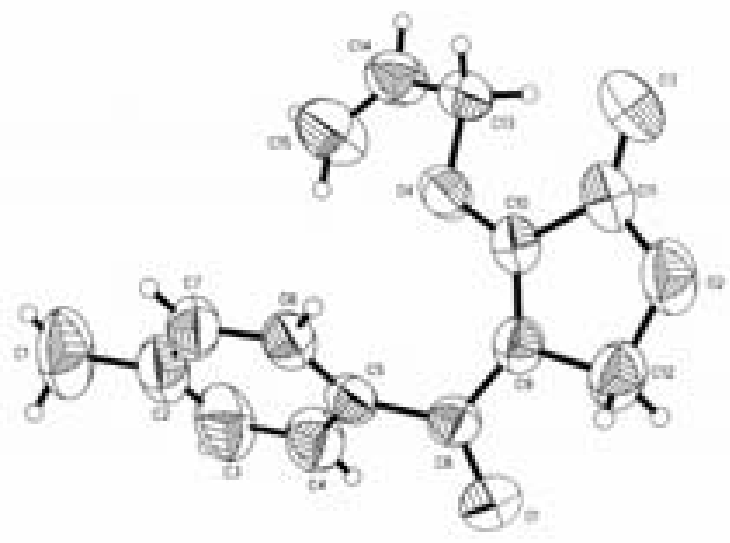

By using this O-alkylation procedure for the preparation of compounds of type 2, a series of diazepines $\mathbf{1}$ were synthesized by reacting several compounds of type $\mathbf{2}\left(\mathrm{R}=\mathrm{CH}_{3}\right)$ with ethylenediamine in chloroform solutions (Table 3). This reaction is not limited to $\mathrm{R}=\mathrm{CH}_{3}$ as demonstrated by the reaction of $\mathbf{2} \mathbf{k}\left(\mathrm{R}=\mathrm{CH}_{2}=\mathrm{CHCH}_{2}-\right)$ with 1,2-ethylenediamine which formed the corresponding diazepine $\mathbf{1 f}$ in $66 \%$ yield (up from $39 \%$ for $\mathbf{2 f}, \mathrm{R}=\mathrm{CH}_{3}$ ).

\section{Conclusions}

We have presented a facile route for the formation of 3-alkoxy-2(5H)-furanones 2 . These compounds are key intermediates in the synthesis of furodiazepines. These furodiazepines possess interesting structural similarities to benzodiazepines which are presently under biological evaluation, and shall be reported elsewhere.

\section{Acknowledgments}

The authors thank the Deutsche Akademische Austauschdient (DAAD) for partial financial support for CPL. JAKB thanks Dr. Alan Pinkerton, Department of Chemistry, University of Toledo for the use of his SMART 2K CCD diffractometer. 


\title{
Experimental
}

\author{
General
}

Melting points were determined on a Melt-Temp apparatus and are uncorrected. TLC was conducted on plated prepared from E. Merck silica gel $60 \mathrm{~F}_{254}, 0.2 \mathrm{~mm}$ thickness. Silica gel from EM science in a column with $20 \mathrm{~mm}$ diameter was used for flash column chromatography pressured with compressed nitrogen. NMR spectra were acquired on a Bruker AC250 spectrometer with TMS as internal standard. A Hewlett-Packard $6890 \mathrm{Gas}$ chromatograph/mass spectrometer was used to record MS data. For high resolution mass spectra a Kratos MS-801 DS-55 spectrometer was used. Elemental analysis were performed by M-H-W Laboratories, Phoenix, Arizona.

General Procedure: 4-Aroyl-3-alkoxy-2(5H)-furanones (2).

DBU (0.37 mL, $2.5 \mathrm{mmol})$ was added to a solution of $3(2.5 \mathrm{mmol})$ in anhydrous DMF (35 $\mathrm{mL})$ in a three-neck-round-bottom flask equipped with a thermometer and a magnetic stirring bar under an inert atmosphere. The solution was cooled to a temperature between $-10-0^{\circ} \mathrm{C}$ and stirred for $10 \mathrm{~min}$. Then an excess of alkyl iodide was added and the solution was stirred for $2 \mathrm{~h}$. The resulting mixture was allowed to come to room temperature and stirring was continued for $24 \mathrm{~h}$. The yellow-brown reaction mixture was poured into ice water $(300 \mathrm{~mL})$ and extracted with ether $(3 \times 25 \mathrm{~mL})$. The organic layers were combined and dried over anhydrous magnesium sulfate and the solvent was evaporated. The residue was chilled overnight. Solids were recrystallized from ethanol, while oils were subjected to column chromatography on silica gel using methylene chloride as eluent. Spectroscopic and analytical data are given in Table 2.

General Procedure: 7-Aryl-4,5-dihydro-2-oxo-3H,8H-furo[3,4-b][1,4]diazepines (1).

To a solution of $2(2.5 \mathrm{mmol})$ in chloroform $(50 \mathrm{~mL})$ was added 1,2-ethylenediamine (3 mmol) under an inert gas atmosphere. The mixture was stirred at room temperature for $24 \mathrm{~h}$. The solvent was evaporated and the resulting residue was recrystallized from methanol or ethanol. Spectroscopic and analytical data are given in Table 3. 
Table 2: ${ }^{1} \mathrm{H}-{ }^{13} \mathrm{C}-\mathrm{NMR}, \mathrm{MS}$ and analytical data of 2a-q and 4

\begin{tabular}{|c|c|c|c|c|c|c|}
\hline Product & $\begin{array}{l}{ }^{1} \mathbf{H} \mathbf{N M R}^{\mathbf{a}} \\
\delta(\mathbf{p p m})\end{array}$ & $\begin{array}{l}{ }^{13} \mathbf{C} \mathbf{N M R}^{\mathrm{a}} \\
\delta(\mathbf{p p m})\end{array}$ & $\begin{array}{c}\text { Molecular } \\
\text { formula }\end{array}$ & MS & \multicolumn{2}{|c|}{$\begin{array}{l}\text { Analysis \% } \\
\text { Calc./Found }\end{array}$} \\
\hline & & & & & C & $\mathbf{H}$ \\
\hline $2 \mathbf{a}$ & $\begin{array}{l}1.17(\mathrm{t}, 3 \mathrm{H}), 4.32(\mathrm{q}, 2 \mathrm{H}), \\
5.01(\mathrm{~s}, 2 \mathrm{H}), 7.50,7.63, \\
7.86(\mathrm{~m}, \mathrm{~m}, \mathrm{~d}, 5 \mathrm{H})\end{array}$ & $\begin{array}{l}14.85,67.63,67.85,127.97, \\
128.18,128.98,133.58, \\
136.32,142.79,167.59, \\
189.49\end{array}$ & $\mathrm{C}_{13} \mathrm{H}_{12} \mathrm{O}_{4}$ & $\begin{array}{l}232,203,188, \\
159,143,132, \\
105\end{array}$ & $\begin{array}{l}67.24 \\
67.30\end{array}$ & $\begin{array}{l}5.01 \\
5.21\end{array}$ \\
\hline $2 \mathbf{b}$ & $\begin{array}{l}4.78(\mathrm{~d}, 2 \mathrm{H}), 5.04(\mathrm{~s}, 2 \mathrm{H}), \\
5.07(\mathrm{~d}, 1 \mathrm{H}), 5.10(\mathrm{~d}, \\
1 \mathrm{H}), 5.76(\mathrm{~m}, 1 \mathrm{H}), 7.50, \\
7.63,7.85(\mathrm{~m}, \mathrm{~m}, \mathrm{~d}, 5 \mathrm{H})\end{array}$ & $\begin{array}{l}67.72,71.74,118.97 \\
128.35,129.15,130.25, \\
131.60,133.74,136.36, \\
143.54,167.67,189.40\end{array}$ & $\mathrm{C}_{14} \mathrm{H}_{12} \mathrm{O}_{4}$ & $\begin{array}{l}244,172,122, \\
105\end{array}$ & $\begin{array}{l}68.85 \\
68.68\end{array}$ & $\begin{array}{l}4.95 \\
5.03\end{array}$ \\
\hline $2 \mathrm{c}$ & $\begin{array}{l}1.01(\mathrm{t}, 3 \mathrm{H}), 4.47(\mathrm{q}, 2 \mathrm{H}) \\
5.05(\mathrm{~s}, 2 \mathrm{H}), 7.40(\mathrm{~m}, 4 \mathrm{H})\end{array}$ & $\begin{array}{l}14.30,66.28,67.20,126.32, \\
127,22,127.89,128.94, \\
130.03,131.05,138.10, \\
146.62,166.95,188.09\end{array}$ & $\mathrm{C}_{13} \mathrm{H}_{11} \mathrm{ClO}_{4}$ & $\begin{array}{l}266,231,203, \\
166,159,139, \\
131\end{array}$ & $\begin{array}{l}58.55 \\
58.65\end{array}$ & $\begin{array}{l}4.16 \\
4.35\end{array}$ \\
\hline $2 d$ & $\begin{array}{l}0.62(\mathrm{~d}, 6 \mathrm{H}), 1.58(\mathrm{~m}, \\
1 \mathrm{H}), 4.22(\mathrm{~d}, 2 \mathrm{H}), 5.06(\mathrm{~s}, \\
2 \mathrm{H}), 7.39(\mathrm{~m}, 4 \mathrm{H})\end{array}$ & $\begin{array}{l}\text { 18.19, 28.44, 66.79, 77.70, } \\
126.87,127.17,128.34, \\
129.65,130.88,131.50, \\
138.89,147.21,167.54, \\
188.85\end{array}$ & $\mathrm{C}_{15} \mathrm{H}_{15} \mathrm{ClO}_{4}$ & $\begin{array}{l}294,239,203 \\
159,139,131\end{array}$ & $\begin{array}{l}61.13 \\
61.19\end{array}$ & $\begin{array}{l}5.13 \\
5.27\end{array}$ \\
\hline $2 e$ & $\begin{array}{l}4.13(\mathrm{~s}, 3 \mathrm{H}), 5.08(\mathrm{~s}, 2 \mathrm{H}), \\
7.62,7.88,8.07(3 \mathrm{~m}, 4 \mathrm{H})\end{array}$ & $\begin{array}{l}59.03,67.14,112.65, \\
117.47,126.59,129.15, \\
132.53,132.77,136.07, \\
137.30,145.31,187.05\end{array}$ & $\mathrm{C}_{13} \mathrm{H}_{9} \mathrm{NO}_{4}$ & $\begin{array}{l}243,214,168, \\
130,102\end{array}$ & $\begin{array}{l}64.20 \\
64.40\end{array}$ & $\begin{array}{l}3.73 \\
3.90\end{array}$ \\
\hline $2 \mathrm{f}$ & $\begin{array}{l}2.38(\mathrm{~s}, 3 \mathrm{H}), 3.87(\mathrm{~s}, 3 \mathrm{H}), \\
4.94(\mathrm{~s}, 2 \mathrm{H}), 7.23(\mathrm{~d}, 2 \mathrm{H}), \\
7.71(\mathrm{~d}, 2 \mathrm{H})\end{array}$ & $\mathrm{b}$ & $\mathrm{C}_{13} \mathrm{H}_{12} \mathrm{O}_{4}$ & $\begin{array}{l}232,189,159, \\
119,91\end{array}$ & $\mathrm{~b}$ & \\
\hline $2 \mathrm{~g}$ & $\begin{array}{l}1.11(\mathrm{t}, 3 \mathrm{H}), 2.35(\mathrm{~s}, 3 \mathrm{H}), \\
4.21(\mathrm{q}, 2 \mathrm{H}), 4.91(\mathrm{~s}, 2 \mathrm{H}), \\
7.21(\mathrm{~d}, 2 \mathrm{H}), 7.70(\mathrm{~d}, 2 \mathrm{H})\end{array}$ & $\begin{array}{l}15.02,21.60,67.84,68.01, \\
128.51,129.11,129.36, \\
133.88,143.37,145.05, \\
167.79,189.19\end{array}$ & $\mathrm{C}_{14} \mathrm{H}_{14} \mathrm{O}_{4}$ & $\begin{array}{l}246,231,189, \\
159,146,119, \\
91\end{array}$ & $\begin{array}{l}68.27 \\
68.52\end{array}$ & $\begin{array}{l}5.73 \\
5.72\end{array}$ \\
\hline $2 \mathrm{~h}$ & $\begin{array}{l}0.80(\mathrm{t}, 3 \mathrm{H}), 1.19 \quad(\mathrm{~m}, \\
2 \mathrm{H}), 1.51(\mathrm{~m}, 2 \mathrm{H}), 2.45 \\
(\mathrm{~s}, 3 \mathrm{H}), 4.27(\mathrm{t}, 2 \mathrm{H}), 5.03 \\
(\mathrm{~s}, 2 \mathrm{H}), 7.29(\mathrm{~d}, 2 \mathrm{H}), 7.77 \\
(\mathrm{~d}, 2 \mathrm{H})\end{array}$ & $\begin{array}{l}13.46,18.56,21.77,31.52, \\
67.97,72.06,128.60, \\
129.19,129.49,134.17, \\
143.91,145.05,167.96, \\
189.39\end{array}$ & $\mathrm{C}_{16} \mathrm{H}_{18} \mathrm{O}_{4}$ & $\begin{array}{l}275(\mathrm{M}+1), \\
219,119,91\end{array}$ & $\begin{array}{l}70.06 \\
70.23\end{array}$ & $\begin{array}{l}6.62 \\
6.60\end{array}$ \\
\hline
\end{tabular}


Table 2 (Cont.)

\begin{tabular}{|c|c|c|c|c|c|c|}
\hline $2 \mathbf{i}$ & $\begin{array}{l}0.85(\mathrm{t}, 3 \mathrm{H}), 1.21(\mathrm{~m}, \\
8 \mathrm{H}), 1.52(\mathrm{~m}, 2 \mathrm{H}), 2.44 \\
(\mathrm{~s}, 3 \mathrm{H}), 4.27(\mathrm{t}, 2 \mathrm{H}), 5.03 \\
(\mathrm{~s}, 2 \mathrm{H}), 7.29(\mathrm{~d}, 2 \mathrm{H}), 7.67 \\
(\mathrm{~d}, 2 \mathrm{H})\end{array}$ & $\begin{array}{l}13.87,21.64,22.36,25.22, \\
28.60,29.41,31.47,67.85, \\
72.19,128.60,129.06, \\
129.40,134.08,143.83, \\
144.85,167.88,189.26\end{array}$ & $\mathrm{C}_{19} \mathrm{H}_{24} \mathrm{O}_{4}$ & $\begin{array}{l}316,301,219 \\
146,119,91\end{array}$ & $\begin{array}{l}72.11 \\
72.20\end{array}$ & $\begin{array}{l}7.65 \\
7.62\end{array}$ \\
\hline $2 \mathbf{j}$ & $\begin{array}{l}2.43(\mathrm{~s}, 3 \mathrm{H}), 5.02(\mathrm{~s}, 2 \mathrm{H}) \\
5.39(\mathrm{~s}, 2 \mathrm{H}), 7.10(\mathrm{~m}, \\
2 \mathrm{H}), 7.25(\mathrm{~m}, 5 \mathrm{H}), 7.69 \\
(\mathrm{~d}, 2 \mathrm{H})\end{array}$ & $\begin{array}{l}21.81,68.01,72.95,127.88, \\
127.97,128.43,128.51, \\
129.23,129.58,130.58, \\
135.18,143.29,145.02, \\
168.05,189.10\end{array}$ & $\mathrm{C}_{19} \mathrm{H}_{16} \mathrm{O}_{4}$ & $\begin{array}{l}308,278,225, \\
187,144,119, \\
91\end{array}$ & $\begin{array}{l}74.00 \\
74.06\end{array}$ & $\begin{array}{l}5.23 \\
5.33\end{array}$ \\
\hline $2 k$ & $\begin{array}{l}2.37(\mathrm{~s}, 3 \mathrm{H}), 4.76(\mathrm{~d}, 2 \mathrm{H}), \\
4.97(\mathrm{~s}, 2 \mathrm{H}), 5.07(\mathrm{bs}, \\
1 \mathrm{H}), 5.12(\mathrm{bs}, 1 \mathrm{H}), 5.64- \\
5.80(\mathrm{~m}, 1 \mathrm{H}), 7.19(\mathrm{~d}, \\
2 \mathrm{H}), 7.71(\mathrm{~d}, 2 \mathrm{H})\end{array}$ & $\begin{array}{l}21.60,67.80,71.77,118.98, \\
129.10,129.40,129.61 \\
131.68,133.78,142.94 \\
144.97,167.72,188.89\end{array}$ & $\mathrm{C}_{15} \mathrm{H}_{14} \mathrm{O}_{4}$ & $\begin{array}{l}258,243,146 \\
119,91\end{array}$ & $\begin{array}{l}69.74 \\
69.92\end{array}$ & $\begin{array}{l}5.47 \\
5.52\end{array}$ \\
\hline 21 & $\begin{array}{l}0.76(\mathrm{~d}, 6 \mathrm{H}), 1.81(\mathrm{~m}, \\
1 \mathrm{H}), 2.44(\mathrm{~s}, 3 \mathrm{H}), 4.07(\mathrm{~d}, \\
2 \mathrm{H}), 5.04(\mathrm{~s}, 2 \mathrm{H}), 7.28(\mathrm{~d}, \\
2 \mathrm{H}), 7.77(\mathrm{~d}, 2 \mathrm{H})\end{array}$ & $\begin{array}{l}18.52,21.81,28.65,67.97 \\
78.22,128.77,129.24, \\
129.52,134.21,144.17 \\
145.01,168.05,189.45\end{array}$ & $\mathrm{C}_{16} \mathrm{H}_{18} \mathrm{O}_{4}$ & $\begin{array}{l}274,259,219, \\
174,146,119, \\
91\end{array}$ & $\begin{array}{l}67.83^{c} \\
67.59\end{array}$ & $\begin{array}{r}6.76^{\mathrm{c}} \\
6.34\end{array}$ \\
\hline $2 m$ & $\begin{array}{l}1.28(\mathrm{t}, 3 \mathrm{H}), 2.43(\mathrm{~s}, 3 \mathrm{H}), \\
4.23(\mathrm{q}, 2 \mathrm{H}), 5.01(\mathrm{~s}, 2 \mathrm{H}), \\
5.08(\mathrm{~s}, 2 \mathrm{H}), 7.27(\mathrm{~d}, 2 \mathrm{H}), \\
7.88(\mathrm{~d}, 2 \mathrm{H})\end{array}$ & $\begin{array}{l}14.01,21.73,61.60,65.90, \\
68.01,129.15,129.70, \\
133.79,141.89,144.93, \\
167.71,168.26,188.67\end{array}$ & $\mathrm{C}_{16} \mathrm{H}_{16} \mathrm{O}_{6}$ & $\begin{array}{l}305(\mathrm{M}+1), \\
277,185,123, \\
119\end{array}$ & $\begin{array}{l}63.14 \\
63.30\end{array}$ & $\begin{array}{l}5.30 \\
5.25\end{array}$ \\
\hline $2 n$ & $\begin{array}{l}1.16(\mathrm{~d}, 6 \mathrm{H}), 2.45(\mathrm{~s}, 3 \mathrm{H}), \\
5.05(\mathrm{~s}, 2 \mathrm{H}), 5.25(\mathrm{qu}, \\
1 \mathrm{H}), 7.27(\mathrm{~d}, 2 \mathrm{H}), 7.89 \\
(\mathrm{~d}, 2 \mathrm{H})\end{array}$ & $\begin{array}{l}21.73,22.45,67.97,74.93, \\
128.94,129.61,130.29, \\
133.88,143.08,144.85, \\
168.22,189.28\end{array}$ & $\mathrm{C}_{15} \mathrm{H}_{16} \mathrm{O}_{4}$ & $\begin{array}{l}261(\mathrm{M}+1), \\
219,147,119, \\
91\end{array}$ & $\begin{array}{l}69.20 \\
68.94\end{array}$ & $\begin{array}{l}6.20 \\
6.10\end{array}$ \\
\hline 20 & $\begin{array}{l}1.39-1.45(\mathrm{~m}, 4 \mathrm{H}), 1.60 \\
-1.66(\mathrm{~m}, 4 \mathrm{H}), 2.45(\mathrm{~s} \\
3 \mathrm{H}), 5.06(\mathrm{~s}, 2 \mathrm{H}), 5.48(\mathrm{t}, \\
1 \mathrm{H}), 7.28(\mathrm{~d}, 2 \mathrm{H}), 7.76 \\
(\mathrm{~d}, 2 \mathrm{H})\end{array}$ & $\begin{array}{l}21.69,23.12,33.12,67.93, \\
84.26,128.85,129.44, \\
130.04,134.04,143.07 \\
144.63,168.14,189.27\end{array}$ & $\mathrm{C}_{17} \mathrm{H}_{18} \mathrm{O}_{4}$ & $\begin{array}{l}286,219,119, \\
91\end{array}$ & $\begin{array}{l}71.31 \\
71.36\end{array}$ & $\begin{array}{l}6.34 \\
6.29\end{array}$ \\
\hline $2 p$ & $\begin{array}{l}4.13(\mathrm{~s}, 3 \mathrm{H}), 5.09(\mathrm{~s}, 2 \mathrm{H}) \\
7.27(\mathrm{~m}, 2 \mathrm{H}), 8.34(\mathrm{~m}, \\
2 \mathrm{H})\end{array}$ & $\begin{array}{l}59.13,67.31,123.41, \\
126.59,129.95,141.44, \\
145.78,150.20,167.14, \\
187.73\end{array}$ & $\mathrm{C}_{12} \mathrm{H}_{9} \mathrm{NO}_{6}$ & $\begin{array}{l}263,146,150 \\
104\end{array}$ & $\begin{array}{l}54.76 \\
54.77\end{array}$ & $\begin{array}{l}3.45 \\
3.21\end{array}$ \\
\hline
\end{tabular}


Table 2 (Cont.)

\begin{tabular}{|c|c|c|c|c|c|c|}
\hline $2 q$ & $\begin{array}{l}2.59(\mathrm{~s}, 3 \mathrm{H}), 4.07(\mathrm{~s}, 3 \mathrm{H}), \\
5.01(\mathrm{~s}, 2 \mathrm{H}), 6.89(\mathrm{~d}, 1 \mathrm{H}), \\
7.71(\mathrm{~d}, 1 \mathrm{H})\end{array}$ & $\mathrm{b}$ & $\mathrm{C}_{11} \mathrm{H}_{10} \mathrm{O}_{4} \mathrm{~S}$ & $\begin{array}{l}238,223,140, \\
125,112,97\end{array}$ & $b$ & \\
\hline 4 & $\begin{array}{l}2.41(\mathrm{~s}, 6 \mathrm{H}), 4.98(\mathrm{~s}, 4 \mathrm{H}) \\
5.08(\mathrm{~s}, 4 \mathrm{H}), 7.04(\mathrm{~m}, \\
2 \mathrm{H}), 7.19(\mathrm{~d}, 4 \mathrm{H}), 7.62 \\
(\mathrm{~m}, 2 \mathrm{H}), 7.77(\mathrm{~d}, 4 \mathrm{H})\end{array}$ & $\begin{array}{l}21.65,67.88,70.28,128.79, \\
129.11,129.28,130.61, \\
133.79,142.95,145.02, \\
167.67\end{array}$ & $\mathrm{C}_{32} \mathrm{H}_{26} \mathrm{O}_{8}$ & $\begin{array}{l}539(\mathrm{M}+1), \\
321,195,119\end{array}$ & $\begin{array}{l}71.37 \\
71.47\end{array}$ & $\begin{array}{l}4.87 \\
4.97\end{array}$ \\
\hline
\end{tabular}

a) in $\mathrm{CDCl}_{3}$ as solvent, $\mathrm{q}=$ quartet, qu= quintet b) not determined c) calcd. for $\mathrm{C}_{16} \mathrm{H}_{18} \mathrm{O}_{4} \bullet 0.5 \mathrm{H}_{2} \mathrm{O}$

Table 3: ${ }^{1} \mathrm{H}-\mathrm{NMR}$, MS and analytical data of 1c,e,f,p and q

\begin{tabular}{|c|c|c|c|c|c|c|c|c|}
\hline \multirow[t]{2}{*}{ Product $^{\mathrm{a}}$} & \multirow[t]{2}{*}{$\begin{array}{c}\text { Yield } \\
(\%)\end{array}$} & \multirow[t]{2}{*}{$\begin{array}{l}\mathrm{Mp} \\
\left({ }^{\circ} \mathbf{C}\right)\end{array}$} & \multirow[t]{2}{*}{$\begin{array}{c}{ }^{1} \mathbf{H} \mathbf{N M R}^{\mathbf{b}} \\
\delta(\mathbf{p p m})\end{array}$} & \multirow[t]{2}{*}{$\begin{array}{c}\text { Molecular } \\
\text { formula }\end{array}$} & \multirow[t]{2}{*}{ MS } & \multicolumn{3}{|c|}{$\begin{array}{l}\text { Analysis \% } \\
\text { Calc./Found }\end{array}$} \\
\hline & & & & & & $\mathbf{C}$ & $\mathbf{H}$ & $\mathbf{N}$ \\
\hline 1c & 42 & 176 & $\begin{array}{l}3.55(\mathrm{bs}, 2 \mathrm{H}), 3.94(\mathrm{bs}, 2 \mathrm{H}), \\
4.56(\mathrm{bs}, 2 \mathrm{H}), 6.39(\mathrm{bs}, 1 \mathrm{H}), \\
7.37(\mathrm{~m}, 4 \mathrm{H})\end{array}$ & $\mathrm{C}_{13} \mathrm{H}_{11} \mathrm{ClN}_{2} \mathrm{O}_{2}$ & $\begin{array}{l}262,227, \\
217,183\end{array}$ & $\begin{array}{l}59.44 \\
59.54\end{array}$ & $\begin{array}{l}4.22 \\
4.27\end{array}$ & $\begin{array}{l}10.66 \\
10.82\end{array}$ \\
\hline 1e & 48 & 172 & $\begin{array}{l}3.62(\mathrm{bs}, 2 \mathrm{H}), 4.21(\mathrm{bs}, 2 \mathrm{H}) \\
4.76(\mathrm{~s}, 2 \mathrm{H}), 5.68(\mathrm{bs}, 1 \mathrm{H}) \\
7.55,7.74(2 \mathrm{~m}, 4 \mathrm{H})\end{array}$ & $\mathrm{C}_{14} \mathrm{H}_{11} \mathrm{~N}_{3} \mathrm{O}_{2}$ & $\begin{array}{l}253,252 \\
208,181 \\
179,142 \\
140,102\end{array}$ & $\begin{array}{l}66.40 \\
66.60\end{array}$ & $\begin{array}{l}4.38 \\
4.60\end{array}$ & $\begin{array}{l}16.59 \\
16.68\end{array}$ \\
\hline 1f & 39 & 178 & $\begin{array}{l}2.38(\mathrm{~s}, 3 \mathrm{H}), 3.61(\mathrm{bs}, 2 \mathrm{H}), 4.20 \\
(\mathrm{bs}, 2 \mathrm{H}), 4.78(\mathrm{~s}, 2 \mathrm{H}), 5.31(\mathrm{bs} \\
1 \mathrm{H}), 7.22(\mathrm{~m}, 2 \mathrm{H}), 7.34(\mathrm{~m}, 2 \mathrm{H})\end{array}$ & $\mathrm{C}_{14} \mathrm{H}_{14} \mathrm{~N}_{2} \mathrm{O}_{2}$ & $\begin{array}{l}242,227 \\
197,183 \\
170,128 \\
105,91\end{array}$ & $\begin{array}{l}69.41 \\
69.22\end{array}$ & $\begin{array}{l}5.82 \\
5.90\end{array}$ & \\
\hline $1 p$ & 51 & 198 & $\begin{array}{l}3.57(\mathrm{bs}, 2 \mathrm{H}), 4.15(\mathrm{bs}, 2 \mathrm{H}), \\
4.64(\mathrm{~s}, 2 \mathrm{H}), 5.51(\mathrm{bs}, 1 \mathrm{H}), 7.53 \\
(\mathrm{~d}, 2 \mathrm{H}), 8.17(\mathrm{~d}, 2 \mathrm{H})\end{array}$ & $\mathrm{C}_{13} \mathrm{H}_{11} \mathrm{~N}_{3} \mathrm{O}_{4}$ & $\begin{array}{l}273,256, \\
228,182, \\
151\end{array}$ & $\begin{array}{l}57.14 \\
57.24\end{array}$ & $\begin{array}{l}4.06 \\
4.14\end{array}$ & $\begin{array}{l}15.38 \\
15.31\end{array}$ \\
\hline $1 q$ & 27 & 170 & $\begin{array}{l}2.47(\mathrm{~s}, 3 \mathrm{H}), 3.69(\mathrm{bs}, 2 \mathrm{H}), \\
4.13(\mathrm{bs}, 2 \mathrm{H}), 5.10(\mathrm{~s}, 2 \mathrm{H}), 5.72 \\
(\mathrm{bs}, 1 \mathrm{H}), 6.71(\mathrm{~d}, 1 \mathrm{H}), 7.29(\mathrm{~d}, \\
1 \mathrm{H})\end{array}$ & $\mathrm{C}_{12} \mathrm{H}_{12} \mathrm{~N}_{2} \mathrm{O}_{2} \mathrm{~S}$ & $\begin{array}{l}248,233 \\
215,203 \\
176,111\end{array}$ & $\begin{array}{l}58.05 \\
57.91\end{array}$ & $\begin{array}{l}4.87 \\
5.08\end{array}$ & $\begin{array}{l}11.28 \\
11.27\end{array}$ \\
\hline
\end{tabular}

a) Numbers are given so that Ar will match the ones in Table 1; b) in $\mathrm{CDCl}_{3}$ as solvent 


\section{References and Notes}

1. Zimmer, H.; Nauss, J. L.; Amer, A. J. Heterocyclic.Chem. 1998, 35, 25-28.

2. Pigeon, P.; Decroix, B. Tetrahedron Lett. 1998, 39, 8659-8662 and references cited therein.

3. Fray, M. J.; Bull, D. J.; Cooper, K.; Parry, M. J.; Slefaniak, H. M. J. Med. Chem. 1995, 38, 35243535.

4. Kawakami, Y.; Kitani, H.; Yuasa, S.; Abe, M.; Moriwaki, M.; Kagoshima,M.; Tersawa, M.; Tahara, T. Eur. J. Med. Chem..1996, 31, 683-692.

5. Rajappan, V. P.; Hosmane, R. S. Nucleosides Nucleotides 1998, 17, 1141-1151.

6. Bertelli, L.; Biagi, G.; Giorgi, I.; Livi, O.; Manera, C. Farmaco 1998, 53, 305-311.

7. Goerlitzer, K.; Wilpert, C.; Ruebsamen-Waigmann, H.; Suhartono, H; Wang, L.; Immelmann, A. Arch. Pharm. (Weinheim Ger.) 1995, 328, 247-255.

8. Zimmer, H.; Amer, A.; Ho, D.; Palmer-Sungail, R. J. Heterocyclic Chem. 1991, 28, 1501-1510.

9. Amer, A.; Ventura, M.; Zimmer, H. J. Heterocyclic Chem. 1983, 20, 359-364 and references cited therein.

10. Zimmer, H.; Palmer-Sungail, R.; Ho, D.; Amer, A. J. Heterocyclic Chem. 1993, 30, 161-167.

11. Crystallographic data for the structures reported in this paper have been deposited with Cambridge Crystallographic Data Centre as supplementary publications no. CCDC 146275146279. These data can be obtained free of charge via www.ccdc.cam.ac.uk/conts/retrieving.html (or from the CCDC, 12 Union Road, Cambridge CB2 1EZ, UK; fax: +44 1223 336033; e-mail: deposit@ccdc.cam.ac.uk).

Sample Availability: Not Available

(C) 2003 by MDPI (http://www.mdpi.org). Reproduction is permitted for noncommercial purposes. 